\title{
Impact of breastfeeding on infant and child mortality with varying levels of immunization and HIVIAIDS: Evidence from 2008/09 Kenya Demographic and Health Survey
}

\author{
Boniface Omuga K'Oyugi \\ Population Studies and Research Institute, University of Nairobi, Kenya
}

\section{Email address:}

bkoyugi@uonbi.ac.ke

\section{To cite this article:}

Boniface Omuga K'Oyugi. Impact of Breastfeeding on Infant and Child Mortality with Varying Levels of Immunization and HIV/AIDS: Evidence from 2008/09 Kenya Demographic and Health Survey. Social Sciences. Vol. 3, No. 1, 2014, pp. 9-16.

doi: $10.11648 /$ j.ss.20140301.13

\begin{abstract}
Appropriate breastfeeding is one of the cost effective measures in the broader strategy for Integrated Management of Childhood Illness (IMCI) Programme which was adopted by the international community in the 1990s to reduce infant and child mortality especially in developing countries such as Kenya. Although Kenya experienced great improvements towards attainment of full immunization coverage in 1990s and early 2000s, it also experienced high HIV and malaria prevalence rates in some parts of the country during the two decades. This paper used the 2008/09 Kenya Demographic and Health Survey data to analyze the impact of breastfeeding duration in the face of varying levels of immunization coverage and HIV/AIDS risk perception. A total of 6,079 births born 59 months or less preceding the survey were used in the analysis. Life table and generalized linear regression in log linear format were the main methods applied. To determine the breastfeeding effect, other broad community level contextual and socio-economic as well as individual level biological and household environmental factors were controlled for in the analysis. Births in communities with longer breastfeeding duration exceeding 13 months had about 34 per cent lower infant and child mortality when compared with their other counterparts. Longer breastfeeding duration was also found to have beneficial effect on child survival even in lower immunization and higher HIV/AIDS risk situations. Multiple births and those born to mother in widowed/divorced/separated marital status had undesired effect on child mortality. The study results underscores the need for strengthening aspects of the IMCI Programme aimed at promoting breastfeeding and immunization of children as stipulated in the Ministry of Health guidelines which are also in line with those provided by the World Health Organization (WHO) and the United Programme on HIV/AIDS (UNAIDS). Further research is also necessary to explain the mechanism and key proximate determinants through which breastfeeding and immunization act to strongly influence childhood mortality in Kenya.
\end{abstract}

Keywords: Breastfeeding, Immunization, HIV/AIDS, Infant Child Mortality, Kenya, Demographic and Health Survey

\section{Introduction}

In 2000, the World Health Organization released a special bulletin on Child Mortality in which analysis of factors explaining the 1990 s trends in childhood mortality in developing countries were discussed in detail [1]. One of the principal concerns was birth spacing and breastfeeding factors that had not improved sufficiently so as to reduce childhood mortality substantially. It was also observed that lapses in efforts especially in seeking of medical attention for respiratory illnesses and reduced efforts to immunize children could lead to slowing of and even reversal of decline in mortality rates. A closer look at Kenya's childhood mortality rates from data gathered since 1998 indicate that the country had a reversal of mortality decline in 1998-2003 and a substantial mortality decline in 2003-2008. The aim of this study was to examine more critically the impact of breastfeeding factor in Kenya especially in the face of varying levels of immunization and HIV/AIDS.

Scientific evidence supports strongly the importance of breastfeeding factor in promoting healthy development of children. Breast milk: helps to protect against infectious diseases; is associated with improved cognitive development; contains anti-bodies against many organisms both viral and bacterial hence helps in protecting the child against immune related diseases like tetanus, haemophilus 
pertussis, polio, and coxsackie virus [2]. Breastfeeding is exclusive when a child receives only breast milk and no other food or a drink supplement including water. On the other hand, full breastfeeding is when a child receives other fluids such as juice or water. According to UNAIDS Guidelines, "when replacement feeding is acceptable, feasible, affordable, sustainable and safe, avoidance of all breastfeeding by HIV infected mothers is recommended, otherwise exclusive breastfeeding is recommended during the first months of life" and "anti-retroviral therapy to mother and infant is likely to offer possibility of maintaining breastfeeding as a safe option for HIV infected women" [3]. Islamic literature also stress on two years of breastfeeding as being good for both the mother and child from a wide range of aspects [4].

In Kenya, earlier studies established that children breastfed for relatively longer duration experienced significantly reduced mortality rates when compared with their counterparts breastfed for shorter durations $[5,6]$. A recent study using data from Delta State in Nigeria concluded that lower levels of less than 50 per cent immunization coverage and 10 per cent of exclusive breastfeeding were not important determinants of child mortality [3]. This implies that there are minimum threshold levels of immunization coverage and breastfeeding duration before protective benefits can be realized. Available literature also suggests that in some developing countries the impact of breastfeeding is greatly modified by socioeconomic and household environmental factors. In Gueni-Bissau, the risk of death of weaned children increased to 3.5 times when adjusted for maternal education and ethnic group and that excess mortality was independent of age at weaning [7]. In Malaysia, adjusted risks of death due to not breastfeeding were: 5.2 if the household had neither a toilet nor piped water; 2.67 if the household had a toilet only; 2.51 if the household had both a toilet and piped water; and, 21 percent of all deaths were due to not breastfeeding [8]. More recent studies concluded that the determinants are modified by removing barriers pertaining to: health care delivery systems such as quality of services referral services and logistics [9]; health care personnel such as adequacy, training and attitude of health care workers [10]; community members such as beliefs, customs and practices especially with respect to adopting modern technology [11].

\section{Data and Methodology}

\subsection{Source and Description of Data}

The study data was obtained from the 2008/09 Kenya Demographic and Health Survey (KDHS). Analysis was restricted to 6,079 births born 59 months or less from the date of interview. Women in the reproductive age group 15 to 49 years living in sampled urban and rural households in all regions of Kenya were targeted. The survey data was collected from $13^{\text {th }}$ November 2008 to late February 2009 by the Kenya National Bureau of Statistics (KNBS) in collaboration with other national institutions and with support from ICF Macro. The KDHS data set contain scrambled sampled household and cluster codes to maintain confidentiality of HIV/AIDS information. Survey district codes were available and the study therefore used district as the unit representing a community.

Information in the KDHS data set pertaining to a total of 20 variables was included at the preliminary stage of analysis. The choice of the 20 variables was based on reviewed literature on socio-economic and proximate determinants of infant and child mortality in the analytical framework developed by Mosley and Chen [12]. In addition, the findings of review work by Rutstein [1] were also used. The study focus was the three variables namely breastfeeding, immunization and HIV/AIDS but the other 17 variables were being considered for inclusion in the analysis model as control variables. Information on breastfeeding duration for last closed births was used to compute mean length of breastfeeding duration at community level. Vaccine immunization information for all living children in the sampled households aged 12 to less than 60 months was used to compute full immunization coverage rate at community level. Table 1 provides description of births in terms of percentage distribution and number by categorical variable.

\subsection{Methodology}

The study applied life table analysis and generalizes linear regression methods. Life table method was used to compute infant and child mortality rates. Regression analysis was used to determine breastfeeding effect taking into account other important determinants of infant and child mortality. Statistical Package for Social Sciences (SPSS) was used to compute the required number of child deaths and exposure months. A brief description of procedures used to compute community level estimates of breastfeeding duration, immunization coverage and HIV/AIDS risk are discussed in section 2.2.1. The generalized linear regression model applied is discussed in section 2.2.2.

\subsubsection{Procedures for Computation of Breastfeeding, Immunization and HIV/AIDS Risk}

KDHS data set contains values of breastfeeding durations for individual last closed births. Using the SPSS package, these individual breastfeeding lengths were aggregated at district level and mean lengths by district obtained. The district mean breastfeeding lengths obtained were in-turn assigned to each individual birth in the survey district. The $60^{\text {th }}$ and $40^{\text {th }}$ percentile rule was applied to classify individual mean length value as "Shorter" or "Longer" category.

In order to incorporate information on immunization status of children for children with and without health cards, the method developed by Boerma and others in 1990 was applied [13]. Computation of full immunization coverage 
rate was extended to cover 12 to less than 60 months because the proportion of births alive aged 12 to less than 24 months was found to be very small and would have easily biased the estimates. The $60^{\text {th }}$ and $40^{\text {th }}$ percentile rule was then applied to the individual scores for full immunization coverage to classify them into "Higher" or "Lower" category.

Information in the KDHS dataset on HIV/AIDS risk perception by individual women interviewed was used to compute community level HIV/AIDS risk by aggregating and obtaining district level proportion of those in the category "High risk". The distribution of the obtained values were in-turn subjected to the $60^{\text {th }}$ and $40^{\text {th }}$ percentile rule to classify individual value into "Lower" or "Higher" risk category The other community level variables were computed using this procedure.

Table 1. Percent distribution of births by study variables.

\begin{tabular}{|c|c|c|c|c|c|}
\hline Variable & $\%$ & $\mathbf{N}$ & Variable & $\%$ & $\mathbf{N}$ \\
\hline Age Interval (in months) & & & Marital status & & \\
\hline Under 1 & 3.9 & 237 & Widow/divorce/separate & 8.5 & 515 \\
\hline 1 - under 3 & 4.2 & 257 & Never married & 6.3 & 383 \\
\hline 3 - under 6 & 5.8 & 353 & Married & 85.2 & 5181 \\
\hline 6 -under 12 & 11.5 & 699 & Mother's education & & \\
\hline 12 -under 24 & 19.3 & 1172 & None/primary incomplete. & 52.9 & 3215 \\
\hline $24-$ under 60 & 55.3 & 3361 & Primary complete & 24.9 & 1515 \\
\hline Breastfeeding duration & & & Secondary + & 22.2 & 1349 \\
\hline Shorter & 41.1 & 2498 & Access to information & & \\
\hline Longer & 58.9 & 3581 & Less access & 31.6 & 1923 \\
\hline Full immunization & & & More access & 66.9 & 4067 \\
\hline Lower & 40.0 & 2434 & Birth type & & \\
\hline Higher & 60.0 & 3645 & Single & 97.0 & 5895 \\
\hline HIV/AIDS risk & & & Multiple & 3.0 & 184 \\
\hline Low/Moderate & 41.9 & 2547 & Sex & & \\
\hline High & 58.1 & 3532 & Male & 51.6 & 3134 \\
\hline Malaria endemicity & & & Female & 48.4 & 2945 \\
\hline Low & 46.1 & 2801 & Preceding birth interval & & \\
\hline Endemic/epidemic & 53.9 & 3278 & Shorter & 18.2 & 1104 \\
\hline Nutritional status & & & Longer & 81.8 & 4975 \\
\hline Less malnourished & 59.6 & 3623 & Mother's age at birth & & \\
\hline More malnourished & 40.4 & 2456 & Under 20 and $35+$ years & 72.5 & 4408 \\
\hline Medical Care to sick child & & & 20 - under 35 years & 27.0 & 1644 \\
\hline Less appropriate & 41.0 & 2494 & Birth order & & \\
\hline More appropriate & 59.0 & 3585 & $1^{\text {st }}$ and $7^{\text {th }}$ & 34.4 & 2090 \\
\hline Wealth Index & & & $2^{\text {nd }}$ to $6^{\text {th }}$ & 65.6 & 3989 \\
\hline $1^{\text {st }}$ and $2^{\text {nd }}$ quintiles & 47.0 & 2856 & Source of drinking water & & \\
\hline $3^{\text {rd }}$ quintile & 16.2 & 985 & Non improved & 48.4 & 2941 \\
\hline $4^{\text {th }}$ and $5^{\text {th }}$ quintiles & 36.8 & 2238 & Improved & 49.6 & 3018 \\
\hline Place of residence & & & Main floor material & & \\
\hline Rural & 75.9 & 4612 & Natural/rudimentary & 66.0 & 4014 \\
\hline Urban & 24.1 & 1467 & Finished & 32.5 & 1974 \\
\hline \multicolumn{6}{|l|}{ Type of toilet facility } \\
\hline Non improved & 74.4 & 4523 & & & \\
\hline Improved & 24.1 & 1467 & & & \\
\hline
\end{tabular}

\subsubsection{Brief Description of Generalized Linear Regression Model}

The study used generalized linear regression model in log linear format for count data. The model was deemed suitable since it allows for the dependent variable to have non-normal distribution while at the same time assuming that the dependent variable is linearly related to the explanatory factors or covariates. The model also assumes that the cases are independent observations. In applying this model, the dependent variable was defined as death counts in the age interval and their statistical distribution was assumed to fit a poisson distribution. Logarithmic function was used to perform the required transformation of the dependent variable for purposes of statistical estimation of model parameters. In this regard, the logarithm of age interval exposure months was used as an offset variable in the model specification. Equation 1 provided below is the statistical formula depicting poisson distribution. 


$$
\mathrm{f}(\mathrm{t} \mid \mathrm{x})=\theta_{\mathrm{x}}^{-1} \exp \left(-\mathrm{t} \backslash \theta_{\mathrm{x}}\right) \quad, \mathrm{t}>0
$$

Where

$\theta \mathrm{x}=\mathrm{E}(\mathrm{T} \mid \mathrm{x})$ and a more functional form that is easy to use is $\theta x=\exp (X \beta)$

$X=\left(x_{1} \ldots \ldots . x_{p}\right) \quad$ vector of regression variables

$\mathrm{B}=\left(\beta_{1} \ldots \ldots \ldots \beta_{\mathrm{p}}\right)$ vector of regression coefficients

$\mathrm{E}=$ expectation or mean of the statistical distribution

$\mathrm{T}=$ life time exponentially distributed

$\mathrm{t}=$ failure time

$\mathrm{p}=$ number of regression variables

A logarithmic transformation is applied to the functional equation to obtain the generalized log linear regression model that was used in this study. The mathematical expression of the regression model is provided in Equation 2 below.

$$
\begin{gathered}
\log \left(\theta_{\mathrm{x}}\right)=\beta_{0}+\beta_{1} \mathrm{x}_{1}+\beta_{2} \mathrm{x}_{2}+\ldots+\beta_{\mathrm{p}} \mathrm{x}_{\mathrm{p}} \quad, \text { where } \\
\text { the intercept term }
\end{gathered}
$$

\section{Results and Discussion}

\subsection{Infant and Child Mortality Differentials by Breastfeeding, Immunization Coverage and HIV/AIDS Risk}

Estimates of infant and child mortality rates by the three variables of study interest are provided in Table 2. As per the expectation, longer breastfeeding duration was found to be associated with lower mortality rates in all the six age segments. The beneficial role of higher full immunization coverage in mortality reduction was more dominant from age six months. Similarly, higher mortality rates associated with high HIV/AIDS risk were more pronounced from age 12 months.

The estimates also indicate that most variable categories that were expected to be associated with higher mortality risk had indeed without exception experienced higher rates than the national average. The computed estimates for infant mortality and under-five mortality rates of 51 and 77 per 1,000 live births respectively were fairly close to the official figures contained in the 2008/09 KDHS Report of 52 and 74 per 1,000 live births respectively[14]. The small difference could be attributed to use of un-weighted data in this analysis and difference in reference period. This study used births born 59 months or less from the interview date in the household while methodology used to compute childhood mortality rates presented in the KDHS report used the reference period defined as "births since January 2003".

\begin{tabular}{|c|c|c|c|c|c|c|}
\hline \multirow{2}{*}{ Variable / Category } & \multicolumn{6}{|c|}{ Probability of dying by exact age in months (per 1,000 live births) } \\
\hline & 1 & 3 & 6 & 12 & 24 & 60 \\
\hline \multicolumn{7}{|c|}{ Breastfeeding duration } \\
\hline Shorter & 0.039 & 0.048 & 0.057 & 0.068 & 0.087 & 0.098 \\
\hline Longer & 0.022 & 0.026 & 0.032 & 0.040 & 0.049 & 0.061 \\
\hline \multicolumn{7}{|c|}{ Immunization coverage } \\
\hline Higher & 0.031 & 0.036 & 0.040 & 0.049 & 0.059 & 0.068 \\
\hline \multicolumn{7}{|l|}{ HIV/AIDS risk } \\
\hline Low/Moderate & 0.029 & 0.034 & 0.040 & 0.047 & 0.055 & 0.062 \\
\hline High & 0.028 & 0.036 & 0.045 & 0.058 & 0.077 & 0.097 \\
\hline National average & 0.028 & 0.035 & 0.042 & 0.051 & 0.064 & 0.076 \\
\hline
\end{tabular}

Table 2. Infant and Child Mortality Rates by Breastfeeding Duration, Immunization Coverage and HIV/AIDS Risk.

\begin{tabular}{|c|c|c|c|c|c|c|}
\hline \multirow{2}{*}{ Variable / Category } & \multicolumn{6}{|c|}{ Probability of dying by exact age in months (per 1,000 live births) } \\
\hline & 1 & 3 & 6 & 12 & 24 & 60 \\
\hline \multicolumn{7}{|c|}{ Shorter breastfeeding duration } \\
\hline Lower full immunization & 0.038 & 0.058 & 0.071 & 0.085 & 0.111 & 0.126 \\
\hline Higher full immunization & 0.039 & 0.043 & 0.050 & 0.059 & 0.074 & 0.083 \\
\hline Lower HIV/AIDS risk & 0.040 & 0.048 & 0.054 & 0.061 & 0.074 & 0.081 \\
\hline Higher HIV/AIDS risk & 0.037 & 0.049 & 0.062 & 0.077 & 0.103 & 0.121 \\
\hline \multicolumn{7}{|c|}{ Longer breastfeeding duration } \\
\hline Lower full immunization & 0.018 & 0.023 & 0.029 & 0.039 & 0.053 & 0.068 \\
\hline Higher full immunization & 0.025 & 0.029 & 0.034 & 0.041 & 0.046 & 0.056 \\
\hline Lower HIV/AIDS risk & 0.021 & 0.025 & 0.031 & 0.037 & 0.042 & 0.048 \\
\hline Higher HIV/AIDS risk & 0.022 & 0.027 & 0.033 & 0.045 & 0.059 & 0.080 \\
\hline National average & 0.028 & 0.035 & 0.042 & 0.051 & 0.064 & 0.076 \\
\hline
\end{tabular}

Table 3. Infant and Child Mortality Rates by Breastfeeding Duration with varying levels of Immunization Coverage and HIV/AIDS Risk. 
Table 3 provides further results of mortality rates by breastfeeding duration with varying levels of immunization coverage and HIV/ AIDS risk. The results demonstrate that even in situation of shorter breastfeeding durations, higher immunization coverage and lower HIV/AIDS risk were associated with lower mortality risks. Similarly, in situation of longer breastfeeding duration with higher HIV/AIDS risk and low immunization coverage the study results show that they were generally associated with higher mortality rates. However, age pattern of childhood mortality observed not to be perfect for the case of immunization variable.

\subsection{Impact of Breastfeeding on Infant and Child Mortality}

Results of the final generalized linear regression model fitted to obtain impact of breastfeeding duration on infant and child mortality are provided in Table 4. Before fitting the final model, "univariate" regression analysis incorporating the variable age interval was undertaken for each of the other initial 19 explanatory variables. This was done to control for effect of age pattern on infant and child mortality. At this level of analysis: (i) all the six community level variables were retained to proceed to sub-modeling stage because of their study importance despite the fact that immunization, HIV/AIDS medical care variables were found to be statistically insignificant at 90 percent confidence level; (ii) all the five socio-economic variables were found to be statistically significant at 95 percent confidence level hence retained to proceed to sub-modeling stage; and, only one out of the eight proximate determinants was found to be statistically insignificant hence source of drinking water was dropped at this stage.

At the second stage of sub-modeling, a total of three models were ran and the outcomes were as follows: (i) only nutrition variable was not retained for inclusion in full model since it was found to be both statistically insignificant and highly correlated with other variables in the equation; (ii) information source and place of residence variables were not retained for inclusion in full model since they were found to be either highly statistically insignificant and or not measuring what was intended from the theoretical perspective; and, (iii) three variables namely preceding birth interval, mother's age at birth and birth order were not retained for inclusion in full model since they were found to be highly statistically insignificant. The final model dropped the variable main floor material as it was found to be highly correlated with the variable wealth index. The final model therefore had a total of eleven variables consisting of the three variables of interest to the study and eight other variables serving as controls.

Table 4. Parameter estimates of impact of breastfeeding, immunization, HIV/AIDS risk and other control on infant and child mortality in Kenya.

\begin{tabular}{|c|c|c|c|c|c|c|c|c|c|c|c|c|}
\hline \multirow{2}{*}{ Parameter } & \multicolumn{4}{|c|}{ Model I } & \multicolumn{4}{|c|}{ Model II } & \multicolumn{4}{|c|}{ Model III } \\
\hline & $\boldsymbol{\beta}$ & SE & Sig. & $\mathrm{e}^{\beta}$ & $\boldsymbol{\beta}$ & SE & Sig. & $e^{\beta}$ & $\boldsymbol{\beta}$ & SE & Sig. & $e^{\beta}$ \\
\hline (Intercept) & -0.363 & 0.134 & $0.007^{* * *}$ & 0.696 & -0.415 & 0.200 & $0.038^{* *}$ & 1.515 & 1.683 & 0.266 & $0.000^{* * *}$ & 5.380 \\
\hline \multicolumn{13}{|c|}{ Age Interval (in months) } \\
\hline $1-$ under 3 & -1.993 & 0.191 & $0.000 * * *$ & 0.136 & -1.981 & 0.187 & $0.000^{* * *}$ & 0.138 & -1.898 & 0.186 & $0.000 * * *$ & 0.150 \\
\hline 3 - under 6 & -2.259 & 0.187 & $0.000^{* * *}$ & 0.104 & -2.247 & 0.182 & $0.000 * * *$ & 0.106 & -2.183 & 0.181 & $0.000 * * *$ & 0.113 \\
\hline 6 - under 12 & -2.749 & 0.171 & $0.000^{* * *}$ & 0.064 & -2.766 & 0.167 & $0.000 * * *$ & 0.063 & -2.702 & 0.167 & $0.000 * * *$ & 0.067 \\
\hline $12-$ under 24 & -3.097 & 0.161 & $0.000 * * *$ & 0.045 & -3.125 & 0.158 & $0.000^{* * *}$ & 0.044 & -3.094 & 0.158 & $0.000 * * *$ & 0.045 \\
\hline 24 -under 60 & -4.601 & 0.240 & $0.000 * * *$ & 0.010 & -4.726 & 0.237 & $0.000^{* * *}$ & 0.009 & -4.751 & 0.236 & $0.000 * * *$ & 0.009 \\
\hline Under $1[\mathrm{RC}]$ & 0.000 & . & . & 1.000 & 0.000 & . & . & 1.000 & 0.000 & . & . & 1.000 \\
\hline \multicolumn{13}{|c|}{ Mean length of breastfeeding duration } \\
\hline Longer & -0.391 & 0.116 & $0.001 * * *$ & 0.676 & -0.429 & 0.113 & $0.000 * * *$ & 0.651 & -0.413 & 0.112 & $0.000 * * *$ & 0.662 \\
\hline Shorter $[R C]$ & 0.000 & . & . & 1.000 & 0.000 & . & . & 1.000 & 0.000 & . & . & 1.000 \\
\hline \multicolumn{13}{|c|}{ Full immunization coverage rate } \\
\hline Higher & -0.221 & 0.110 & $0.045 * *$ & 0.802 & -0.252 & 0.110 & $0.022^{* *}$ & 0.777 & -0.287 & 0.109 & $0.009^{* * *}$ & 0.751 \\
\hline Lower $[\mathrm{RC}]$ & 0.000 & . & . & 1.000 & 0.000 & . & & 1.000 & 0.000 & . & . & 1.000 \\
\hline \multicolumn{13}{|l|}{ HIV/AIDS risk } \\
\hline Low /moderate & -0.149 & 0.127 & 0.242 & 0.861 & -0.140 & 0.126 & 0.263 & 0.869 & -0.102 & 0.125 & 0.415 & 0.903 \\
\hline High $[R C]$ & 0.000 & . & . & 1.000 & 0.000 & . & . & 1.000 & 0.000 & . & . & 1.000 \\
\hline \multicolumn{13}{|c|}{ Malaria endemicity } \\
\hline Low & -0.130 & 0.131 & 0.322 & 0.878 & -0.128 & 0.129 & 0.321 & 0.880 & -0.143 & 0.128 & 0.263 & 0.866 \\
\hline
\end{tabular}



HIV/AIDS: Evidence from 2008/09 Kenya Demographic and Health Survey

\begin{tabular}{|c|c|c|c|c|c|c|c|c|c|c|c|c|}
\hline \multirow{2}{*}{ Parameter } & \multicolumn{4}{|c|}{ Model I } & \multicolumn{4}{|c|}{ Model II } & \multicolumn{4}{|c|}{ Model III } \\
\hline & $\boldsymbol{\beta}$ & SE & Sig. & $e^{\beta}$ & $\beta$ & SE & Sig. & $e^{\beta}$ & $\beta$ & SE & Sig. & $e^{\beta}$ \\
\hline $\begin{array}{l}\text { Endemic / epidemic } \\
{[\mathrm{RC}]}\end{array}$ & 0.000 & & & 1.000 & 0.000 & . & & 1.000 & 0.000 & . & . & 1.000 \\
\hline \multicolumn{13}{|c|}{ Medical care to sick children } \\
\hline Appropriate & -0.065 & 0.113 & 0.566 & 0.937 & -0.048 & 0.112 & 0.668 & 0.953 & -0.116 & 0.111 & 0.296 & 0.890 \\
\hline In-appropriate $[\mathrm{RC}]$ & 0.000 & & & 1.000 & 0.000 & . & . & 1.000 & 0.000 & . & . & 1.000 \\
\hline \multicolumn{13}{|l|}{ Wealth index } \\
\hline $3^{\text {rd }}$ quintile & & & & & 0.245 & 0.143 & $0.086^{*}$ & 1.278 & 0.264 & 0.142 & $0.063^{*}$ & 1.303 \\
\hline $4^{\text {th }}$ and $5^{\text {th }}$ quintiles & & & & & -0.022 & 0.136 & 0.871 & 0.978 & 0.034 & 0.141 & 0.811 & 1.034 \\
\hline $1^{\text {st }}$ and $2^{\text {nd }}$ quintiles [RC & & & & & 0.000 & . & & 1.000 & 0.000 & . & . & 1.000 \\
\hline \multicolumn{13}{|l|}{ Marital status } \\
\hline Married & & & & & -1.062 & 0.321 & $0.001 * * *$ & 0.346 & -1.031 & 0.318 & $0.001 * * *$ & 0.357 \\
\hline Never married & & & & & -0.842 & 0.157 & $0.000^{* * *}$ & 0.431 & -0.879 & 0.157 & $0.000 * * *$ & 0.415 \\
\hline Widowed/divorced/sepa & ated $[\mathrm{RC}]$ & & & & 0.000 & 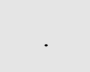 & & 1.000 & 0.000 & . & . & 1.000 \\
\hline \multicolumn{13}{|l|}{ Mother' education level } \\
\hline Secondary + & & & & & -0.026 & 0.134 & 0.845 & 0.974 & -.015 & 0.133 & 0.911 & 0.985 \\
\hline Primary complete & & & & & -0.039 & 0.156 & 0.804 & 0.962 & -.076 & 0.154 & 0.622 & 0.927 \\
\hline None/primary incomple & $\mathrm{e}[\mathrm{RC}]$ & & & & 0.000 & . & . & 1.000 & 0.000 & . & . & 1.000 \\
\hline \multicolumn{13}{|l|}{ Type of birth } \\
\hline Single & & & & & & & & & -1.289 & 0.180 & $0.000 * * *$ & 0.275 \\
\hline Multiple [RC] & . & & & $\cdot$ & & & . & & 0.000 & . & . & 1.000 \\
\hline \multicolumn{13}{|l|}{ Sex } \\
\hline Female & & & & & & & & & -0.054 & 0.106 & 0.607 & 0.947 \\
\hline Male $[\mathrm{RC}]$ & . & & & . & & & 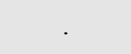 & & 0.000 & . & . & 1.000 \\
\hline \multicolumn{13}{|l|}{ Toilet facility type } \\
\hline Improved & & & & & & & & & -0.033 & 0.142 & 0.819 & 0.968 \\
\hline Non improved [RC] & & & & & & & & & 0.000 & . & . & 1.000 \\
\hline (Scale) & 1.032 & & & & 0.972 & & & & 0.955 & & & \\
\hline $\begin{array}{l}\text { Goodness of fit of } \\
\text { the model }\end{array}$ & Value & df & Sig. & & Value & df & Sig, & & Value & df & Sig. & \\
\hline Log Likelihood & -808.0 & & & & -793.8 & & & & -775.5 & & & \\
\hline Adj. Log Likelihood & -783.2 & & & & -816.9 & & & & -812.1 & & & \\
\hline Likelihood Ratio $\chi^{2}$ & 782.1 & 10 & $0.000 * * *$ & & 859.4 & 16 & $0.000^{* * *}$ & & 912.5 & 19 & $0.000 * * *$ & \\
\hline
\end{tabular}

Key: RC - Reference category; $\beta$ - Regression coefficient; SE - Standard Error; Sig. - Significance; $\mathrm{e}^{\beta}$ - Exponential of $\beta$; df - degrees of freedom; $\quad *$ significant at $90 \%$ confidence level; ** - significant at $95 \%$ confidence level; *** - significant at $99 \%$ confidence level.

Results of the regression analysis are presented in Table 4. Parameter estimates for Models I, II and III in the table are respectively for: sub-model on contextual community level variables; sub-model for community level plus socio-economic variables; and, full model containing community, socio-economic and proximate determinants. It should be noted that only the variables retained for inclusion in the final model were utilized in model building and that the fitting of the final model was guided by the Mosley and Chen framework on analysis of child survival. The study findings are generally consistent with the expectations. Breastfeeding variable was found to have statistically significant reduction impact on infant and child mortality rates even after controlling for immunization, 
HIV/AIDS risk and other important control factors. The key study finding was that longer breastfeeding duration had about 34 per cent reduction effect on infant and child mortality when compared with shorter breastfeeding duration. An interesting observation was that the extremely high statistical significance (99 per cent confidence level) of breastfeeding factor was maintained from the sub-model to the full model stage. This could be interpreted to imply that the breastfeeding effect was fairly strong and remained uncaptured by any of the other socio-economic and proximate variables in the model.

The statistical strength of immunization variable was found to improve from 95 to 99 per cent confidence level at sub-model to final model stage. The finding underscored the beneficial effect of immunization on child survival. In the final model, higher full immunization coverage rates had about 25 per cent reduction effect on infant and child mortality rates relative to lower full immunization coverage. Lower HIV/AIDS risk was found to have statistically insignificant reduction effect on infant and child mortality rates at all stages of model building. This could be interpreted to imply that although lower HIV/AIDS risk perception had reduction effect univariate level, its impact was adequately captured by other variables included in the sub-models and the full model.

As for the other control variables included in the regression analysis age of child, marital status of the mother and type of birth were found to be statistically significant factors effecting infant and child mortality. Births aged under one month, multiple births and those born to widowed/divorced/separated mothers had statistically significant higher infant and child mortality rates when compared with their other respective counterparts. The other observation was that the beneficial effect of improved wealth index appeared to have captured by other proximate variables to the extent that it depicted reverse direction and its effect was statistically insignificant at 95 per cent confidence level. Parameters estimates for model fitness also presented in Table 4 confirm that all the sub-models and the full model were appropriate and statistically significant.

\section{Conclusions and Recommendations}

In this study, life table analysis results established that longer breastfeeding durations of over 13 months was associated with lower infant and child mortality and that mortality differentials by immunization coverage and HIV/AIDS risk were more pronounced after age 12 months. It was further established that even in situations of shorter breastfeeding durations, higher immunization coverage and lower HIV/AIDS risk resulted into reduced infant and child mortality rates. The following four conclusions could be drawn from results of the regression analysis: (i) Longer breastfeeding durations of above 13 months was a significant factor in child survival in Kenya whereby its reduction effect after controlling for other factors was estimated at about 34 per cent.

(ii) The effect of breastfeeding variable was very strong and was not modified as expected by other socio-economic and household environmental factors.

(iii) Immunization had significant reduction effect on infant and child mortality estimated at about 25 per cent after controlling for other factors.

(iv) Lower HIV/AIDS risk was associated with reduced infant and child mortality rates when compared with their counterparts of higher risk as per the expectation although this was found to be statistically insignificant after incorporation of other control factors.

(v) Among the socio-economic and proximate determinant factors, only multiple births and children born to widowed/divorced/separated mothers were found to be statistically significant.

The findings of this study have implications for child survival programmes and for further research. Efforts to improve duration of breastfeeding beyond 13 months and full immunization coverage rates towards universal levels should be intensified so as to sustain reduction of infant and child mortality rates in Kenya. Programme efforts should therefore be made to (i) expand targeted breastfeeding and immunization programme efforts with a focus on enhanced advocacy, information education communication and service delivery; and, (ii) strengthen implementation of programmes addressing mother to child transmission of HIV through breastfeeding and other channels guided by domesticated WHO, UNAIDS protocols.

Based on the socio-economic theory of mortality operationalized by applying the Mosley and Chen analytical model, the effects of breastfeeding and immunization factors were expected to be completely modified by inclusion of proximate determinants in the regression model. Since this was not realized, it means that this study was not able to adequately establish the mechanism through which breastfeeding and immunization factors act to determine infant and child mortality rates in Kenya. In line with the thinking advanced in some of the reviewed studies [9,10,11], there is therefore need for further research that should focus on generation and utilization of more comprehensive data sets with rich information on health services provision so as to be able to incorporate in the analysis variables listed by Rutstein such as barriers to: health care delivery system; health care personnel; and, community beliefs, customs and practices regarding adaptation of modern technology. This in effect implies that greater efforts should be made to promote combined utilization of data generated by Demographic and Health Survey (DHS) and Service Provision Assessment Survey (SPAS) systems. 


\section{References}

[1] S.O. Rutstein, "Factors associated with trends in infant and child mortality in developing countries during the 1990s", Bulletin of the World Health Organization, Special theme Child Mortality, 70 (10), pp. 256-1270, 2000

[2] J. O. Ogbe, "Exclusive breastfeeding and child immunization as demographic determinants of child mortality in Delta State", Pakistan Journal of Nutrition 7 (1), pp. 35-39, 2008.

[3] A. Coutsoudia, "Breastfeeding and HIV positive mother: debate continues", Early Human Development Vol. 81 Issue 1, pp. 87-93, 2005.

[4] S. Balkrishan, "Exploring gender: Islamic perspectives on breastfeeding", International Research Journal of Social Sciences Vol. 2(6), pp. 30-32, 2013.

[5] B. O. K'Oyugi, "The impact of household and community level environmental factors on infant and child mortality in rural Kenya", Unpublished Ph. D thesis, Population Studies and Research Institute, University of Nairobi, pp.163-165, 1992.

[6] P.A. Akwara, "The impact of breastfeeding practices on infant and child mortality in Amagoro Division of Busia, Kenya”, Unpublished MA thesis, Population Studies and Research Institute, University of Nairobi, pp. v-vi, 1994.

[7] K. Molbak et al., "Prolonged breastfeeding, diarrheal disease and survival of children in Guinea-Bissau", Br Med J 308, pp.1403-1406, 1994.

[8] J.P Habicht, J. DaVanzo, W.P. Butz, "Mothers milk and sewerage: their interactive effects on infant mortality", Pediatrics 81(3), pp. 456-461, 1988.
[9] UNICEF, "Levels and trends in child mortality: estimates developed by the UN Inter-Agency Group for child mortality estimation report", http: // www.childinfo.org / mortality.html, cited 2013 June 14.

[10] S. R. B. L. Shrivastava, P.S. Shrivastava and J. Ramasamy, "Infant mortality: need to bridge the existing gap", Sciknow Publications Ltd, International Journal of Maternal and Child Health, I (3), pp.51-52, 2013.

[11] W.M Ramalho, L. M. Serdinha, I. P. Rodrigues and E.C. Duarte, "Inequalities in infant mortality among municipalities in Brazil according to the Family Development Index 2006-2008”, Rev Panam Salud Publice, 33(3), p.205, 2012,

[12] W.H. Mosley and L.C. Chen, "An analytical framework for the study of child survival in developing countries", Population and Development Review, Supplement to Volume 10, pp. 25-45, 1984.

[13] J. T. Boerma, A. E. Sommerfelt, S. O. Rutstein and G. Rojas, "Immunization levels, trends and differential", Demographic and Health Surveys Comparative Studies No. 1: Institute of Resource Development/Macro System Inc., Colombia, Maryland, 1990.

[14] Kenya National Bureau of Statistics (KNBS) and ICF Macro, "Kenya Demographic and Health Survey 2008-09", Calverton, Maryland : KNBS and ICF Macro, p. 104, 2010 